\title{
Multiple drug resistance in salmonellae in England and Wales: a comparison between 1981 and 1988
}

\author{
L R Ward, E J Threlfall, B Rowe
}

\begin{abstract}
Each year from 1981 through to 1988 the most common serotypes isolated from man in England and Wales and identified at the Division of Enteric Pathogens were $S$ typhimurium, $S$ enteritidis, and $S$ virchow. In 1981 these three serotypes accounted for $45 \%, 12 \%$, and $7 \%$ of isolations. The remaining $35 \%$ comprised strains belonging to a further 188 different serotypes, none of which accounted for more than $1 \%$ of the total. In $1988 S$ typhimurium accounted for $24 \%$ of isolations, $S$ enteritidis $57 \%$, and $S$ virchow $4 \%$. The remaining $15 \%$ comprised strains of a further 184 serotypes. The resistances to the common antimicrobial drugs in non-typhoidal salmonellas isolated in England and Wales in 1981 and 1988 were reported with particular reference to resistance to four or more antimicrobial drugs (multiple resistance). For $S$ typhimurium the overall percentage of resistant strains varied little, but multiple resistance more than doubled from $5 \%$ to $12 \%$; in $S$ enteritidis the incidence remained the same. In $S$ virchow the percentages of strains resistant to all the antimicrobial drugs and in particular, to chloramphenicol, streptomycin, trimethoprim and furazolidone, rose from $0.2 \%$ to $10 \cdot 4 \%$.

Salmonella enteritis in man is usually a self limiting disease and antimicrobial treatment is seldom required; but should spread beyond the intestine occur, effective antimicrobial treatment is essential. Under these circumstances a knowledge of the likelihood of resistances to commonly available drugs could be of considerable value to the clinician.
\end{abstract}

Since 1981 the incidence of multiresistant strains isolated from man in England and Wales has doubled for $S$ typhimurium and increased 50-fold for $S$ virchow, but remained constant for $S$ enteritidis. For the remaining serotypes there has been a small overall increase in multiresistant isolates. An upsurge in infections caused by strains belonging to the bovine associated phage types 193 and $204 \mathrm{c}$ has been the main factor responsible for the increased incidence of multiresistant $S$ typhimurium. In contrast, the increase in multiresistant $S$ virchow has been linked to an upsurge in isolations of phage type 19, which is associated with poultry. Multiresistant strains have remained uncommon in $S$ enteritidis. The increase in multiresistant strains of other serotypes is difficult to interpret because of the relatively low numbers involved.

\section{Methods}

Eight thousand, eight hundred and twenty nine non-typhoidal salmonellae isolated from man in England and Wales in 1981 and 26943 strains isolated in 1988 were serotyped and phage typed by appropriate methods. ${ }^{1-4}$ Strains were tested for resistance to antimicrobial drugs by the methods of Anderson and Threlfall ${ }^{5}$ and Frost et al. ${ }^{6}$ These included strip-diffusion tests for resistance to ampicillin (A), chloramphenicol (C), gentamicin (G), neomycin/ kanamycin (K), streptomycin (S) and tetracyclines $(\mathrm{T})$, and spot tests in agar for resistance to sulphonamides ( $\mathrm{Su}$ ), trimethoprim $(\mathrm{Tm})$, furazolidone $(\mathrm{Fu})$ and nalidixic acid $(\mathrm{Nx})$.

For strip-diffusion tests, $80 \mathrm{~mm} \times 7 \mathrm{~mm}$ strips of Ford 428 Mill extra superfine blotting paper were soaked in appropriate antibiotic solutions to give final concentrations per strip of the following (mg/l): A, 200; C, 60; G, 60; $\mathrm{K}, 1000$; S, 500; T, 500. The approximate concentrations at which strains were designated resistant in strip-diffusion tests were as follows (mg/l): A, 20; C, 10; G, 10; K, 50; S, 10; $T, 10$. These concentrations were determined in relation to a panel of antibiotic resistant and antibiotic sensitive salmonellae whose minimum inhibitory concentrations had been previously determined using an agar dilution technique; this panel had been maintained in the culture collection of the Division of Enteric Pathogens. In agar testing the concentrations of antimicrobial drugs incorporated into the plates were as follows (mg/l): $\mathrm{Su}, 100 ; \mathrm{Tm}, 2$; $\mathrm{Fu}, 20 ; \mathrm{Nx}, 40$.

\section{Results}

INCIDENCE OF RESISTANCE

The incidence of resistance in 1981 and 1988 is summarised in table 1.

For $S$ typhimurium, the overall percentages of resistant strains varied little, but the incidence of multiple resistance more than doubled from $5 \%$ in 1981 to $12 \%$ in 1988 .

For $S$ enteritidis, $15 \%$ of strains isolated in 1981 were drug resistant. Because of a dramatic upsurge in this serotype between the end of 1986 and $1988,{ }^{7}$ only $45 \%$ of strains were tested 
for drug resistance. Of those tested, the proportion with resistance was almost identical with that observed in 1981. Compared with $S$ typhimurium and $S$ virchow multiresistance remained rare in $S$ enteritidis (less than $1 \%$ ) and has not changed significantly since 1981 .

For $S$ virchow, the overall incidence of resistance in 1988 was about four times greater than that observed in 1981. Multiresistant strains have shown an even sharper increase from less than $1 \%$ in 1981 to over $10 \%$ in 1988 .

For the remaining serotypes, the incidence of resistance increased slightly from $11 \%$ in 1981 to $17^{\circ} \%$ in 1988 , and the proportion of multiresistant strains from $2 \%$ to $4 \%$. Because of the large number of serotypes involved, however, and the relatively small numbers of strains within each serotype, interpretation is difficult.

\section{RESISTANCE TO INDIVIDUAL ANTIMICROBIAL \\ DRUGS}

A comparison of the incidence of resistance to individual antimicrobial drugs is shown in table 2.

In $S$ typhimurium there have been increases in the incidence of resistance to ampicillin, chloramphenicol, tetracyclines, trimethoprim and nalidixic acid. In particular, the incidence of ampicillin resistance has doubled. The incidence of resistance to streptomycin, sulphonamides, and tetracyclines has remained high in this serotype (more than 14\%) and this has changed little over the eight year period. In contrast, the incidence of resistance to neomycin/kanamycin has dropped from about $6^{\circ}{ }_{0}$ to $1^{\circ}{ }_{0}$. Of particular note is the continuing low incidence of resistance to gentamicin and nalidixic acid (less than $1 \%$ of the total).

In $S$ enteritidis, the incidence of resistance to ampicillin, streptomycin, and tetracyclines increased but, with the exception of furazolidone, resistance to other antimicrobial drugs has shown little change. Resistance to furazolidone ( $20 \mathrm{mg} / \mathrm{l})$ has decreased from $13 \%$ in 1981 to $2 \%$ in 1988.

In $S$ virchow there have been substantial increases in the percentages of strains resistant to all the antimicrobial drugs and, in particular, to chloramphenicol, streptomycin, trimethoprim and furazolidone.

In the remaining serotypes there have been small increases in the incidence of strains resistant to ampicillin, chloramphenicol, neomycin/kanamycin, streptomycin, sulphonamides, tetracyclines, trimethoprim and nalidixic acid.

PATTERNS OF MULTIPLE DRUG RESISTANCE

Multiple resistance was common in $S$ typhimurium and $S$ virchow but rare in $S$ enteritidis. The predominant multiple resistance patterns in the different serotypes are shown in table 3.

In $S$ typhimurium the predominant multiple resistance patterns in 1981 were ACKSSuTTm and ACGKSSuTTm, and in 1988, ASSuT and ACSSuTTm. Phage types in which these patterns were identified included the bovine-associated phage types 204c (ACKSSuTTm in 1981, ACSSuTTm in 1988) and 193 (ASSuT in 1988). Strains isolated in 1981 with the resistance pattern ACGKSSuTTm either did not react with the $S$ typhimurium typing phages (untypable) or belonged to two interrelated phage types, 66 and 122. These two phage types had become rare by 1988 .

In $S$ enteritidis the most common patterns in

Table 1 Incidence of drug resistance in salmonellae isolated in England and Wales in 1981 and 1988

\begin{tabular}{|c|c|c|c|c|c|c|c|c|}
\hline \multirow[b]{2}{*}{ Serotype } & \multirow[b]{2}{*}{ Year } & \multirow[b]{2}{*}{ Number tested } & \multicolumn{2}{|c|}{ Drug resistant } & \multicolumn{4}{|c|}{ Percentage of strains resistant to: } \\
\hline & & & No & $(\%)$ & 1 & 2 & 3 & more than 4 drugs \\
\hline $\begin{array}{l}\text { S typhimurium } \\
\text { S enteritidis } \\
S \text { virchow } \\
\text { Others }\end{array}$ & $\begin{array}{l}1981 \\
1988 \\
1981 \\
1988 \\
1981 \\
1988 \\
1981^{\star} \\
198{ }^{\star}\end{array}$ & $\begin{array}{l}3992 \\
6444 \\
1087 \\
6898^{\star \star} \\
663 \\
1120 \\
3087 \\
3952\end{array}$ & $\begin{array}{r}1454 \\
2069 \\
162 \\
902 \\
106 \\
650 \\
360 \\
690\end{array}$ & $\begin{array}{l}(36 \cdot 4) \\
(32 \cdot 1) \\
(14 \cdot 9) \\
(13 \cdot 1) \\
(16 \cdot 0) \\
(58 \cdot 0) \\
(11 \cdot 7) \\
(17 \cdot 5)\end{array}$ & $\begin{array}{r}6.5 \\
6.8 \\
13.0 \\
7.9 \\
12.9 \\
36.8 \\
5.0 \\
3.2\end{array}$ & $\begin{array}{r}18 \cdot 2 \\
10 \cdot 1 \\
1.0 \\
1.6 \\
2.3 \\
5 \cdot 4 \\
2 \cdot 8 \\
5 \cdot 5\end{array}$ & $\begin{array}{l}6 \cdot 4 \\
4.5 \\
0 \cdot 5 \\
3 \cdot 0 \\
0 \cdot 6 \\
5 \cdot 4 \\
2 \cdot 1 \\
4 \cdot 7\end{array}$ & $\begin{array}{r}5.5 \\
12.3 \\
0.5 \\
0.6 \\
0.2 \\
10.4 \\
1.6 \\
4.0\end{array}$ \\
\hline
\end{tabular}

* 188 serotypes

$\dagger 184$ serotypes
$\star \star 15427$ strains were identified, of which 6898 were tested for resistance to antimicrobial drugs

Table 2 Incidence of resistance to individual antimicrobial drugs in salmonellae isolated in 1981 and 1988

\begin{tabular}{|c|c|c|c|c|c|c|c|c|}
\hline \multirow[b]{3}{*}{ Antimicrobial drug } & \multicolumn{8}{|c|}{ Percentage of strains resistant } \\
\hline & \multicolumn{2}{|c|}{ S typhimurium } & \multicolumn{2}{|c|}{$S$ enteritidis } & \multicolumn{2}{|c|}{$S$ virchow } & \multicolumn{2}{|c|}{ Others } \\
\hline & 1981 & 1988 & 1981 & 1988 & 1981 & 1988 & 1981 & 1988 \\
\hline $\begin{array}{l}\text { Ampicillin } \\
\text { Chloramphenicol } \\
\text { Gentamicin } \\
\text { Neomycin/kanamycin } \\
\text { Streptomycin } \\
\text { Sulphonamides } \\
\text { Tetracyclines } \\
\text { Trimethoprim } \\
\text { Furazolidone } \\
\text { Nalidixic acid }\end{array}$ & $\begin{array}{r}5.4 \\
4.9 \\
<1.0 \\
5.9 \\
16.1 \\
29.7 \\
22.0 \\
8.2 \\
1.5 \\
<1.0\end{array}$ & $\begin{array}{r}11.8 \\
6.1 \\
1.1 \\
1.8 \\
14.5 \\
25.6 \\
23.0 \\
11.0 \\
1.0 \\
<1.0\end{array}$ & $\begin{array}{r}1.3 \\
<1.0 \\
0 \\
1.1 \\
2.4 \\
1.8 \\
1.1 \\
<1.0 \\
13.3 \\
0\end{array}$ & $\begin{array}{r}9.6 \\
<1.0 \\
<1.0 \\
<1.0 \\
4.0 \\
2.1 \\
3.8 \\
<1.0 \\
2.0 \\
0\end{array}$ & $\begin{array}{c}<1.0 \\
<1.0 \\
<1.0 \\
<1.0 \\
<1.0 \\
5.7 \\
1.0 \\
0 \\
8.6 \\
0\end{array}$ & $\begin{array}{r}6.3 \\
8.5 \\
<1.0 \\
3.7 \\
12.9 \\
13.5 \\
11.1 \\
9.2 \\
54.9 \\
<1.0\end{array}$ & $\begin{array}{r}2.7 \\
1.9 \\
<1.0 \\
2.0 \\
5.3 \\
6.5 \\
5.4 \\
1.0 \\
0.3 \\
<1.0\end{array}$ & $\begin{array}{r}5 \cdot 1 \\
2.9 \\
<1.0 \\
3.6 \\
10.8 \\
10.7 \\
10 \cdot 1 \\
4.2 \\
0.9 \\
<1.0\end{array}$ \\
\hline
\end{tabular}


Table 3 Predominant patterns of multiple drug resistance in salmonellae isolated in England and Wales in 1981 and 1988

\begin{tabular}{|c|c|c|c|c|}
\hline \multirow[b]{2}{*}{ Serotype } & \multicolumn{2}{|l|}{1981} & \multicolumn{2}{|l|}{1988} \\
\hline & $R-t y p e^{\star}$ & $\% \dagger$ & $R$-type & $\% \dagger$ \\
\hline S typhimurium & $\begin{array}{l}\text { ACKSSuTTm } \\
\text { ACGKSSuTTm } \\
\text { ASSuT }\end{array}$ & $\begin{array}{l}4 \cdot 1 \\
1 \cdot 6 \\
0 \cdot 6\end{array}$ & $\begin{array}{l}\text { ASSuT } \\
\text { ACSSuTTm }\end{array}$ & $\begin{array}{r}13 \cdot 2 \\
8 \cdot 4\end{array}$ \\
\hline$S$ enteritidis & $\begin{array}{l}\text { ACKSSuT } \\
\text { SSuTFu }\end{array}$ & $\begin{array}{l}1 \cdot 8 \\
0.6\end{array}$ & $\begin{array}{l}\text { ASTFu } \\
\text { SSuTFu }\end{array}$ & $\begin{array}{l}0.8 \\
0.7\end{array}$ \\
\hline$S$ virchow & CKTFu & 0.9 & $\begin{array}{l}\text { CSTTmFu } \\
\text { CSSuTTmFu }\end{array}$ & $\begin{array}{l}2 \cdot 2 \\
2 \cdot 0\end{array}$ \\
\hline Others & $\begin{array}{l}\text { KSSuT } \\
\text { ACKSSuT }\end{array}$ & $\begin{array}{l}1.9 \\
1 \cdot 1\end{array}$ & $\begin{array}{l}\text { ACSSuTTm } \\
\text { CKST }\end{array}$ & $\begin{array}{l}2 \cdot 5 \\
2 \cdot 0\end{array}$ \\
\hline
\end{tabular}

$\star$ R-type $=$ drug resistance pattern

tExpressed as a percentage of resistant strains

the few strains with multiple resistance were ACKSSuT in 1981 and ASTFu in 1988. Only five multiply resistant strains out of 1087 identified were recognised in 1981, however; for 1988 the corresponding figure was 38 out of 6898 tested.

In $S$ virchow the most common pattern in 1981 was CKTFu and in 1988 CSTTmFu and CSSuTTmFu. Strains with the CSTTm and CSSuTTmFu patterns belonged to phage type 19 , a phage type associated with poultry.

The most common patterns in strains of other serotypes were KSSuT and ACKSSuT in 1981, and ACSSuTTm and CKST in 1988. Serotypes in which these patterns were identified included $S$ agona, $S$ blockley, $S$ heidelberg, $S$ krefeld, $S$ oranienberg and $S$ Saintpaul.

\section{Discussion}

These results show that $32 \%$ of $S$ typhimurium and $58^{\circ}$ of $S$ virchow isolated from man in 1988 were resistant to at least one of the commonly used antimicrobial drugs. In contrast, only $13^{\circ} \%$ of $S$ enteritidis were drug resistant. The results also show that the incidence of multiresistant $S$ typhimurium has more than doubled since 1981 but has not changed in $S$ enteritidis. Multiresistant strains have also noticeably increased in $S$ virchow. This is of particular concern because $S$ virchow has been reported to cause infections outside the intestine. ${ }^{89}$ Although the incidence of multiresistant strains has doubled in other serotypes, the overall level of multiresistance in 1988 was less than half of that in S typhimurium and in $S$ virchow.

In 1981 the most common multiple resistance patterns in $S$ typhimurium were ACKSSuTTm and ACGKSSuTTm. Almost all strains with the ACKSSuTTm pattern belonged to phage type 204c, which has been the most common phage type causing bovine salmonellosis in England and Wales since 1983. ${ }^{1011}$ Strains with the pattern ACGKSSuTTm either belonged to phage type 66 and 122 or were untypable. Most strains in phage types $66 / 122$ and with this drug resistance combination had been isolated from travellers infected abroad, particularly in the Indian subcontinent. Similar strains are known to have caused extensive outbreaks in that area since $1978 .{ }^{12}$ In 1988 the most common pattern of multiple resistance was ASSuT, and most strains with this pattern belonged to phage type 193. Phage type 193 has been subdivided, ${ }^{10}$ but strains resistant to ASSuT seem to belong to a clone which was first identified in bovines in 1986 (Rowe B and Threlfall EJ, unpublished observations). The second most common pattern in 1988 was ACSSuTTm. Strains with this pattern belonged to a neomycin/ kanamycin-sensitive derivative of phage type 204c which has become widely distributed in cattle since $1983 .{ }^{13}$ The increased incidence of multiresistant $S$ typhimurium has been almost entirely caused by an upsurge of infections caused by these two phage types associated with bovines.

In $198887 \%$ of $S$ enteritidis strains were drug sensitive. Over $80 \%$ of infections were caused by phage type $4,{ }^{7}$ and because the most strains of this phage type were drug sensitive, this is reflected in the low incidence of resistance in $S$ enteritidis. The only resistances that increased significantly were to ampicillin, streptomycin, and tetracyclines. For the most part these increases were caused by the appearance and spread of a strain of $S$ enteritidis phage type 24 resistant to these three antibiotics. ${ }^{14}$

Over $50 \%$ of $S$ virchow isolated in 1988 were resistant to furazolidone (MIC: $>100 \mathrm{mg} / \mathrm{l}$ ). One of the main food animal reservoirs of $S$ virchow is poultry and the high incidence of furazolidone resistance may reflect the use of this drug or a related antimicrobial drug in chickens. Of strains isolated in 1988, $10 \%$ were multiresistant and the most common patterns were CSTTmFu and CSSuTTmFu. Almost all strains with these patterns belonged to PT19. This is phage type associated with poultry that has become more prevalent in man since 1986 (Rowe B, unpublished observations).

In 1981 multiresistant strains were recognised in only 23 of 188 serotypes other than $S$ typhimurium, $S$ enteritidis, and $S$ virchow. In contrast, 43 of 184 serotypes identified in 1988 had multiresistant isolates. There have also been increases in the percentages of multiresistant strains in some of the more common serotypes. This has been particularly noticeable in $S$ agona and $S$ saintpaul, and also in $S$ blockley, a serotype which has shown a three fold increase in incidence since 1981 .

In England and Wales a comparison of blood and faecal salmonella isolates suggests that 
bloodstream infections occur in less than $2 \%$ of cases (Rowe B, unpublished observations). Nevertheless, although systemic salmonellosis is rare, the increasing incidence of salmonella strains with resistance to multiple combinations of commonly used therapeutic antibiotics may present problems in treatment. When choosing treatment before antimicrobial sensitivity results are available, a knowledge of the likelihood of multiple resistance may be useful.

1 Kauffman F. In: Serological diagnosis of salmonella species. Copenhagen: Munksgaard, 1972.

2 Callow B. A new phage-typing scheme for Salmonella typhimurium. J Hyg 1959;57:346-59.

3 Ward LR, de Sa JDH, Rowe B. A phage-typing scheme for Salmonella enteritidis Epidemiol Infect 1978;99:291-4.

4 Chambers RM, McAdam P, de Sa JDH, Ward LR, Rowe B. A phage-typing scheme for Salmonella virchow. FEMS A phage-typing scheme for Saln
Microbiol Lett 1987;40:155-7.
5 Anderson ES, Threlfall EJ. The characterization of plasmids in the enterobacteria. $J$ Hyg 1974;72:471-87.

6 Frost JA, Threlfall EJ, Willshaw GA. Methods of studying transferable resistance to antibiotics in vitro. In: Russel transferable resistance to antibiotics in vitro. In: Russel AP, Quesnel LB, eds. Assessment of antimicrobial activity and resistance. SAB Technical
Academic Press, 1983:265-84.

7 Anonymous. Salmonella in eggs. [Editorial.] PHLS Microbiol Digest 1989;6:1-9.

8 Wilkins EGL, Roberts C. Extraintestinal salmonellosis. Epidemiol Infect 1988;100:361-8.

9 Mandal BK, Brennand J. Bacteraemia in salmonellosis: a 15 year retrospective study from a regional infectious diseases unit. Br Med J 1988:1242-3.

10 Rowe B, Threlfall EJ. Antibiotic resistance in salmonella PHLS Microbiol Digest 1986;3:6-8.

11 Anonymous. Animal salmonellosis 1988. Ministry of Agriculture, Fisheries and Food, Welsh Office Agriculture Department, Department of Agriculture and Fisheries for Scotland.

2 Frost JA, Rowe B, Ward LR, Threlfall EJ. Characterization of resistance plasmids and carried phages in an epidemic clone of multi-resistant Salmonella typhimurium in India J Hyg 1982;88:193-204.

13 Threlfall EJ, Rowe B, Ward LR. Increase in prevalence of a neomycin/kanamycin-sensitive variant of $S$ typhimurium PT204c in cattle in Britain. Vet Rec 1987;15:366-7.

14 Frost JA, Ward LR, Rowe B. Acquisition of a drug resistance plasmid converts Salmonella enteritidis phage type 4 to phage type 24. Epidemiol Infect 1989;103:243-8. 